Digital technologies in

contemporary architecture and the illusion of objectivity

in the design process

\section{| RESUMEN |}

Este artículo intenta poner al descubierto las contradicciones que existen entre los argumentos racionalistas y las arbitrariedades formales que hoy se observan en el uso de tecnologías digitales en la arquitectura contemporánea. Al mismo tiempo, se pretende reconocer y poner en valor a los aspectos subjetivos, arbitrarios y no cuantificables que existen en las decisiones de diseño.

\section{| ABSTRACT |}

This article attempts to expose the contradictions between the rationalist arguments and formal arbitrariness observed today in the use of digital technologies in contemporary architecture. At the same time, it seeks to recognize the value of the subjective, arbitrary and not-quantifiable aspects in design decision-making.

\title{
Tecnologías digitales en la arquitectura contemporánea y la ilusión de objetividad en los procesos de diseño
}

\section{INTRODUCCIÓN}

Una tendencia de vanguardia en la arquitectura contemporánea es la búsqueda de nuevas expresiones formales y materiales derivadas del uso intenso de tecnologías digitales. Su consecuencia más evidente es una profusión global de edificios de geometrías no tradicionales en todas partes del planeta. Las envolventes hipercontinuas, las fachadas paramétricas o las estructuras celulares están presentes, sin distinción, en todo tipo de edificios y programas. Sin embargo, a pesar de su aparente vanguardismo formal, sus discursos conceptuales están todavía profundamente enraizados en ideales modernistas propios del siglo pasado. La obsesión racionalista por objetivizar los procesos de diseño y eliminar cualquier rasgo de arbitrariedad ha derivado hoy en un conjunto de discursos pseudocientíficos y metáforas tecnológicas inconsistentes que evidencian profundas motivaciones estéticas.

Este artículo busca examinar las inconsistencias en los argumentos que intentan racionalizar y objetivizar las arbitrariedades formales derivadas del uso de tecnologías digitales en la arquitectura contemporánea. Asimismo, se pretende reconocer, asumir y poner en valor los aspectos subjetivos y no cuantificables que existen en las decisiones de diseño como una condición propia de la arquitectura.

\section{POSITIVISMO TECNOLÓGICO Y LA OBSESIÓN POR LA OBJETIVIDAD}

El Movimiento Moderno estaba inspirado en una visión de la sociedad fuertemente basada en el conocimiento científico y en la fe ciega en la tecnología como motor de progreso. La consolidación de la industrialización, los triunfos de la ingeniería y los cambios sociales y políticos de posguerra parecían determinar un camino ineludible hacia una sociedad y una arquitectura más funcional y tecnológica. La búsqueda de la racionalidad en el diseño se convirtió en una obsesión. La metáfora de la máquina de habitar resumía sin ambigüedades el anhelo por una arquitectura funcionalista y objetiva.

* Arquitecto de la Universidad de Chile, Master in Architecture and Urbanism por la Architectural Association School of Architecture y Senior Architect en Zaha Hadid Architects. Actualmente es profesor invitado en el Departamento de Arquitectura de la Universidad de Chile.

** Arquitecto de la Universidad de Chile, Master in Design Studies por Harvard University y profesor de CAD/CAM en el Departamento de Arquitectura de la Universidad de Chile. 
1. Revestimiento Wind Kinetic

Guillaume Avelez Larraufie + Clement Lassus + Antoine Maurice Tison

Curso «Revestimiento Contemporáneo», FAU, Prof. Diego Rossel

Aunque el posmodernismo y las tendencias arquitectónicas que le siguieron parecían haber matizado esta visión, el surgimiento de nuevas tecnologías computacionales en esta década ha dejado al descubierto una reaparición explícita del ideario modernista del progreso basado en la técnica. La inmensa cantidad de herramientas digitales, cada vez más ubicuas, completas y poderosas, parecen confirmar la aspiración que la tecnología todo lo puede. La variedad actual de software para arquitectura va desde la simulación energética o acústica hasta el análisis de comportamiento de multitudes, haciendo fácilmente creer que (casi) todas las decisiones de diseño pueden instrumentalizarse y basarse en información cuantitativa objetiva.

Junto con la expansión explosiva del uso de tecnologías digitales, se ha desencadenado también una irrupción de discursos

arquitectónicos cargados de metáforas científicas y referencias positivistas tecnológicas. El software se ha convertido en el vehículo ideal para objetivizar las decisiones de diseño bajo un esquema de causa-efecto. La célebre frase de Sullivan, «la forma sigue a la función», tiene hoy múltiples reinterpretaciones que van desde las metáforas naturalistas hasta referencias hacia los procesos productivos.

Un ejemplo lo constituyen el creciente grupo de edificios con envolventes tapizadas en patrones exuberantes, cuyos discursos típicamente recurren a metáforas científicas o matemáticas de inspiración en la naturaleza: desde autómatas celulares o diagramas de Voronoi, hasta L-Systems o geometrías de fractales. Sin duda, el diseño paramétrico y el scripting han sido claves en facilitar la tarea de generar, distorsionar y aplicar un patrón para obtener un resultado de alta complejidad visual. Pero más allá de la beneficiosa capacidad de cálculo de las herramientas, lo que se argumenta y enfatiza en estos proyectos es la referencia literal entre los algoritmos matemáticos y las operaciones de diseño, invocando a un criterio de neutralidad ajeno al diseñador que permite justificar hasta la más intricada forma resultante. La forma sigue a la naturaleza.

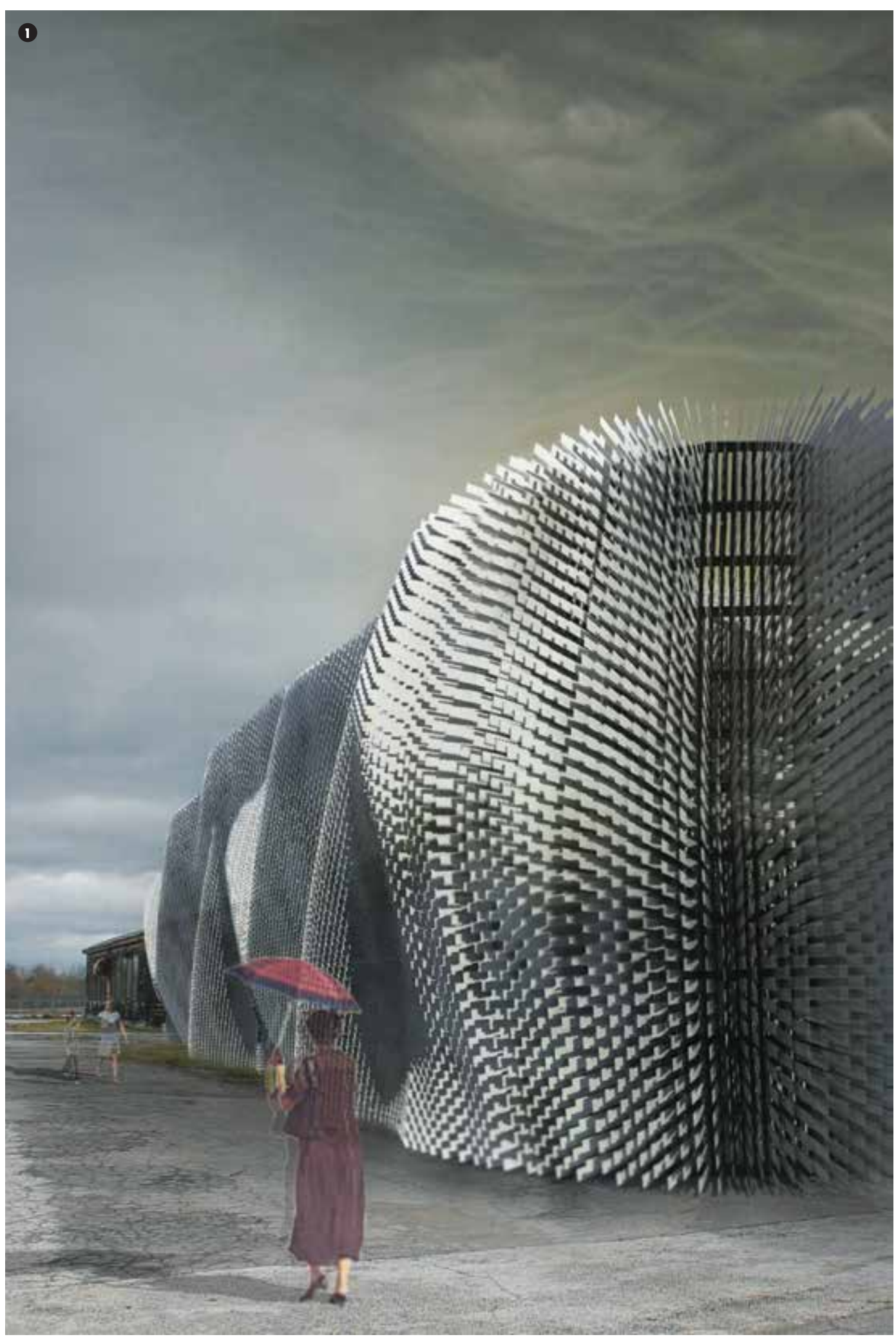


2. Revestimiento Pavo Real

Javiera Alexa Díaz Cabrera + Francisca Ignacia Carrasco Pizarro + Diego Andrés Poblete Letelier

Curso «Revestimiento Contemporáneo», FAU, Prof. Diego Rossel

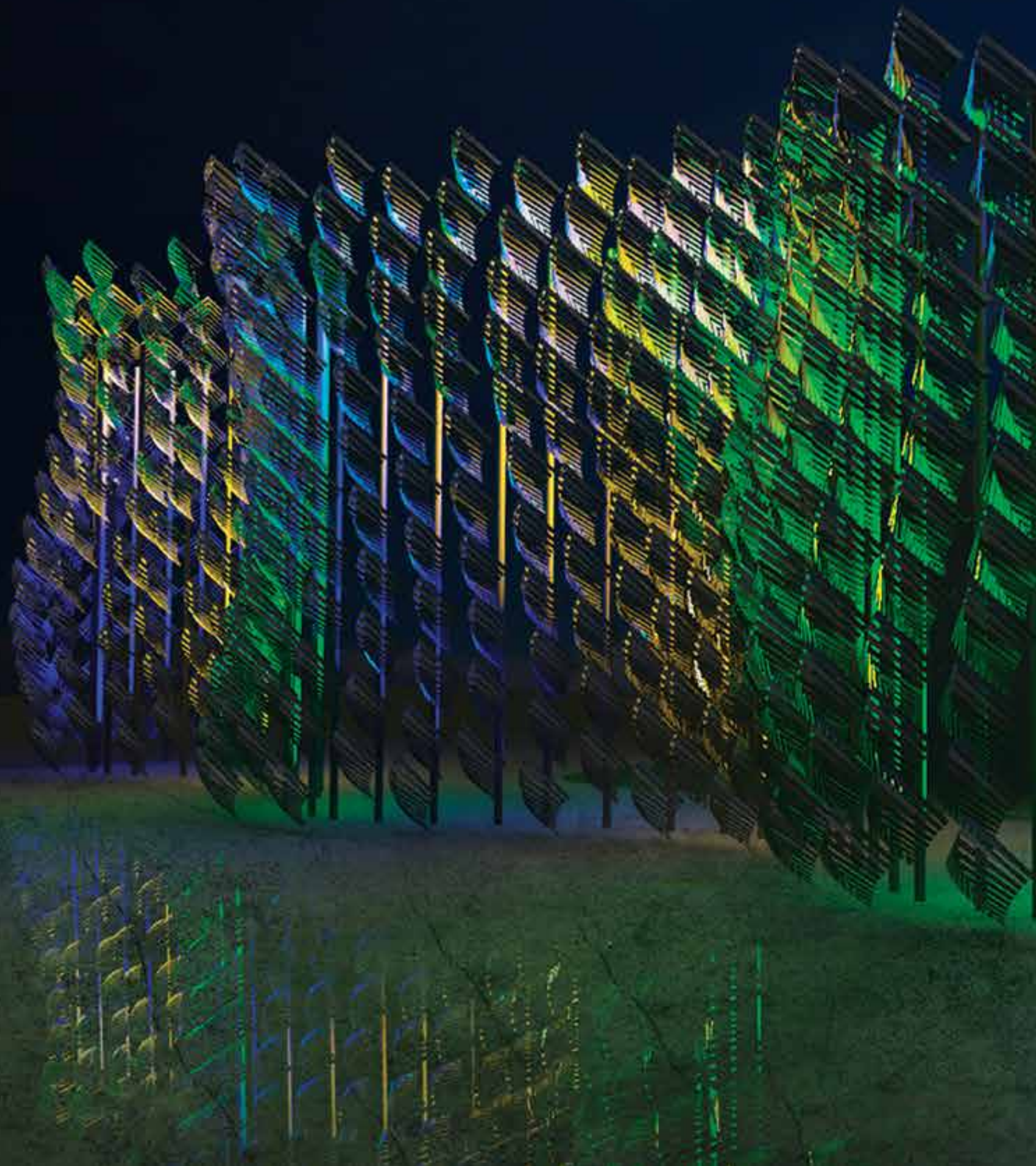


3. Revestimiento en metal

Diego Montoya

Curso «Tecnologías CAD/CAM en Arquitectura», FAU, Prof. Mauricio Loyola
Otro ejemplo similar son aquellas fachadas generadas a partir de cientos de módulos variados que descansan en el ideal de una funcionalidad perfecta: fachadas climáticas que controlan el sol, el ruido, la luz, el viento, o casi cualquier otra cosa. La efervescencia por la sustentabilidad y la moral ecológica de fin de siglo representan el motivo ideal para aludir a criterios de hipereficiencia, optimización y dominio técnico sobre el medio ambiente. Las herramientas de simulación, cada vez más precisas, aparecen hoy como la fuente de validación científica, y por tanto incuestionable, de la arquitectura. Es la doctrina del croquis reemplazado por un gráfico de barras. La forma sigue a la eficiencia.

Un último ejemplo se encuentra en los edificios de volúmenes escultóricos que desafían la geometría cartesiana, usualmente con extravagancia. Indudablemente, su existencia se vio facilitada (factibilizada, más bien) por la masificación de tecnologías CAM y de fabricación digital. En estas obras, la búsqueda de la racionalidad raya en el determinismo tecnológico, casi insinuando que las formas escultóricas son resultado del uso de una determinada máquina. En este discurso, las formas escultóricas constituyen el resultado de la huella «inevitable» de un proceso productivo complejo. Es la reinterpretación contemporánea de las referencias (y reverencias) modernas hacia la industria y la construcción. La complejidad material fabricada digitalmente es, hoy, la textura del moldaje en el hormigón para Le Corbusier o el lenguaje de los detalles constructivos en acero para Mies. La forma sigue a la fabricación.

Sin embargo, es de sentido común observar que en todos estos casos, a pesar de las insistentes referencias de objetividad y racionalidad técnica, los diseños están cargados de arbitrariedad y subjetividad. Un autómata celular puede responder a un modelo matemático totalmente objetivo, pero la sola decisión de usarlo en una fachada es una decisión tremendamente subjetiva. Una fachada climática («piel»o «membrana», en la manía de las metáforas científicas) puede aparecer como la «solución» (otro concepto esencialmente determinista) más

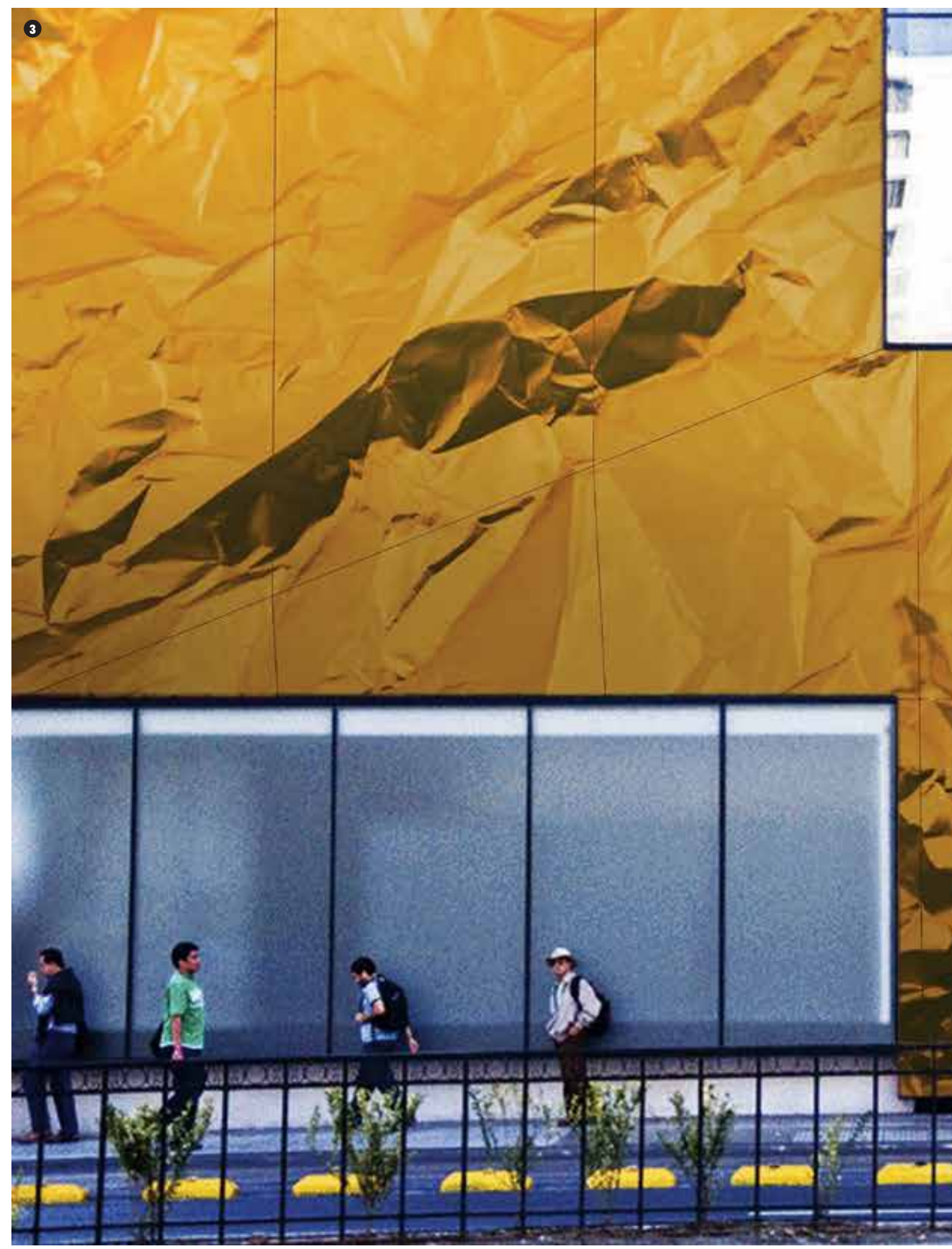




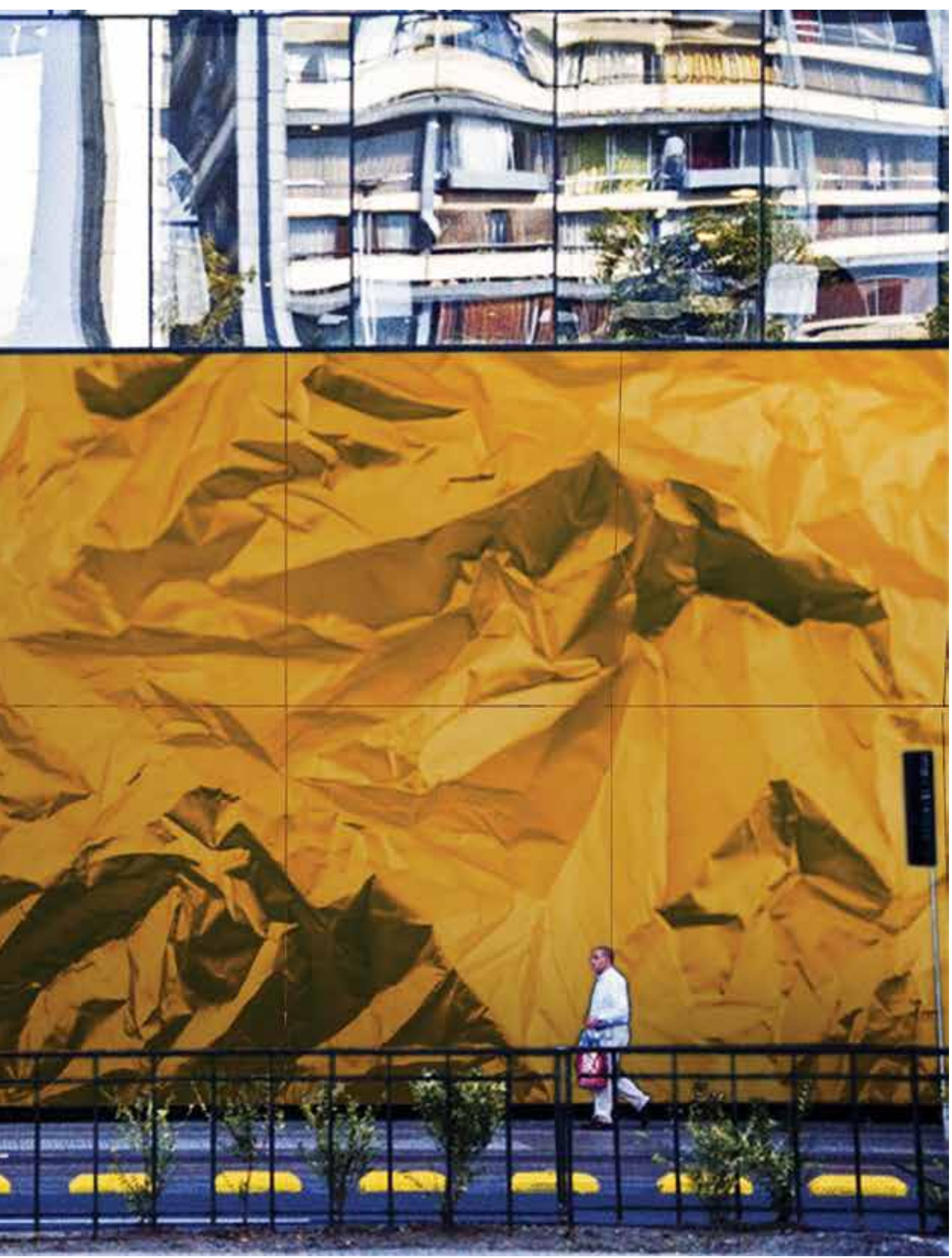

óptima para el control solar, pero en el fondo, toda la cadena de decisiones de diseño es profundamente arbitraria.

\section{ARBITRARIEDAD Y SUBJETIVIDAD EN LA ARQUITECTURA CONTEMPORÁNEA}

La contradicción es evidente. Pese a la supuesta objetividad existente en los procesos proyectuales, los resultados -las obras- están afectadas por decisiones y preferencias estéticas arbitrarias. El síntoma más obvio es la existencia de múltiples rasgos formales comunes, constituyendo un lenguaje de diseño claramente identificable, que es compartido, validado y replicado por sus creadores. Aunque se arguyen múltiples y diferentes procesos de diseño «objetivos» y «no arbitrarios», todos los resultados tienden a la reiteración de las mismas características formales. Para Patrick Schumacher, esto es tan evidente y reconocible que incluso debiera ser considerado como un estilo arquitectónico. La aspiración modernista de eliminar la arbitrariedad de los procesos de diseño, aparentemente lograda con la racionalidad del diseño paramétrico o la fabricación digital, no es tal: ni el software ni una máquina $\mathrm{CNC}$ tienen preferencias estéticas.

Lo cierto es que la arbitrariedad y la subjetividad son rasgos inherentes a nuestra condición humana, y por tanto, al diseño y la arquitectura. Las preferencias, obsesiones, deseos y pretensiones de los arquitectos son imposibles de reprimir, y tarde o temprano, se manifiestan en sus obras. Dentro de estas, la búsqueda del placer estético juega un papel significativo en arquitectura, aunque se intente disminuirla, contenerla o simplemente disimularla.

La arbitrariedad en las decisiones de diseño, las predilecciones y obstinaciones estéticas personales están presentes en todos los proyectos, y reconocer su existencia no es más que sincerar nuestra propia individualidad. Sin embargo, cuando esto ocurre, surge inmediatamente la visión modernista para censurarla. La enorme crítica que despierta la obra de Frank Gehry o Zaha Hadid es 
4. Ornamento en madera

Sofía Caro

Curso «Tecnologías CAD/CAM en Arquitectura», FAU, Prof. Mauricio Loyolo

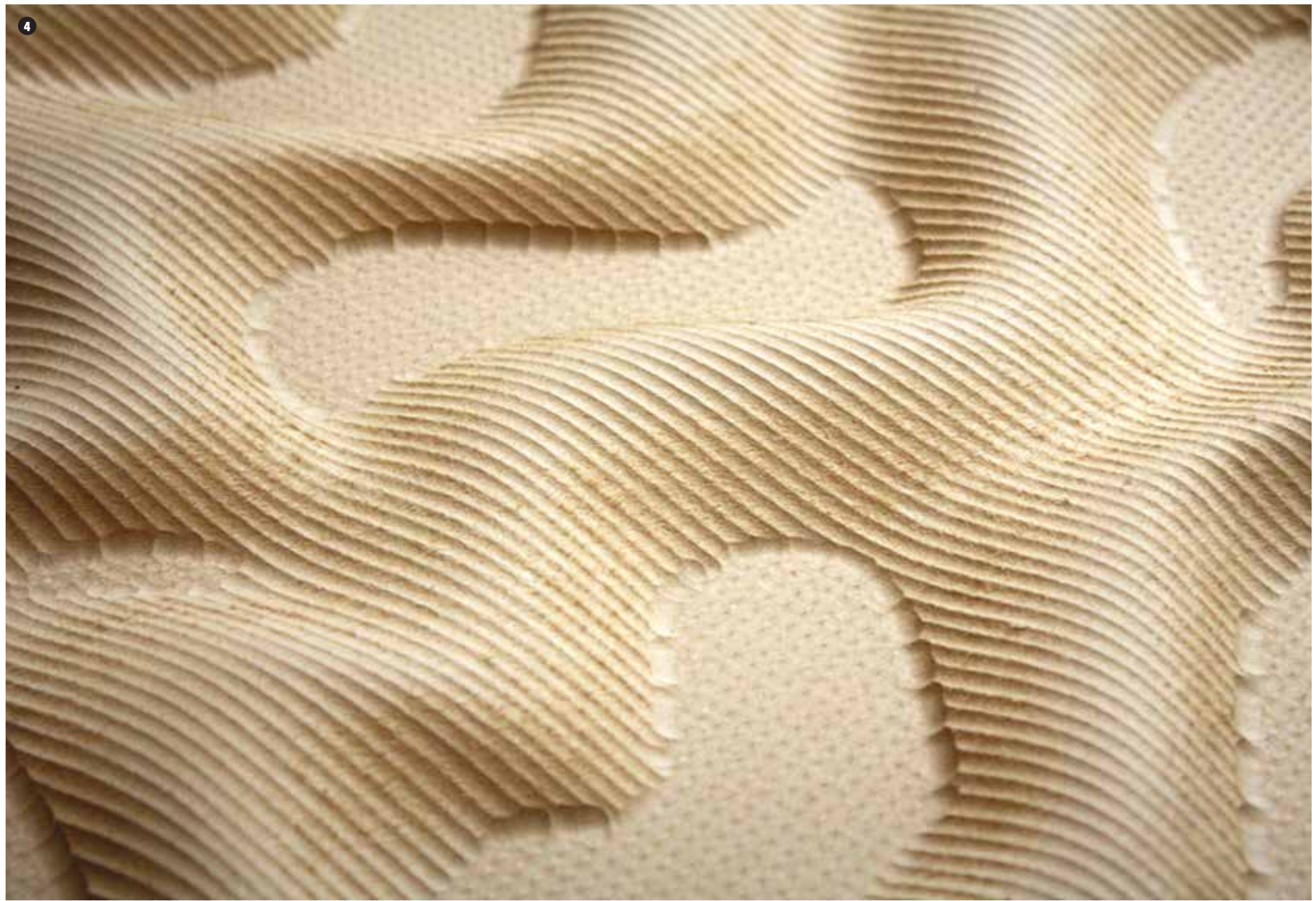

testimonio de ello. Caprichosa, innecesaria, excéntrica; absolutamente contraria al ideal de la forma eficiente, neutra, funcional, objetiva. Irónicamente, cuando las mismas formas vienen acompañadas de una memoria de inspiración científica o de motivación tecnológica, entonces se entiende validada. La popularización de los diagramas de proceso es evidencia de esta necesidad patológica por la explicación racional.

La represión modernista de la arbitrariedad ha levantado críticas en reiteradas ocasiones.
Robert Venturi en los sesenta, Bernard Tschumi en los setenta, James Stirling en los ochenta o Juhani Pallasmaa en los noventa, son solo algunos casos entre quienes se han rebelado en contra del discurso funcionalista y tecnocéntrico. Cada uno desde una vereda diferente, estos autores comparten la visión de abordar el problema arquitectónico desde la complejidad, la disyunción y la contradicción, entendiendo que estos conceptos son características fundamentales de la disciplina. Conciben al sujeto como un agente dinámico, complejo e impredecible que toma decisiones en base a sus creencias, experiencias y dimensión cultural.

Reconocer la subjetividad, asumir la arbitrariedad y liberar las decisiones arquitectónicas de sus ataduras causalistas permite abrir camino a nuevas oportunidades para la arquitectura. Surge el espacio para revisitar algunas de las preguntas que fueron olvidadas o reprimidas por la modernidad. La búsqueda (o la erradicación) del ornamento es uno de los ejemplos paradigmáticos de esta condición. 
5. Unidad de habitación de Marsella, Le Corbusier Fotografía: Mircea Tuncan

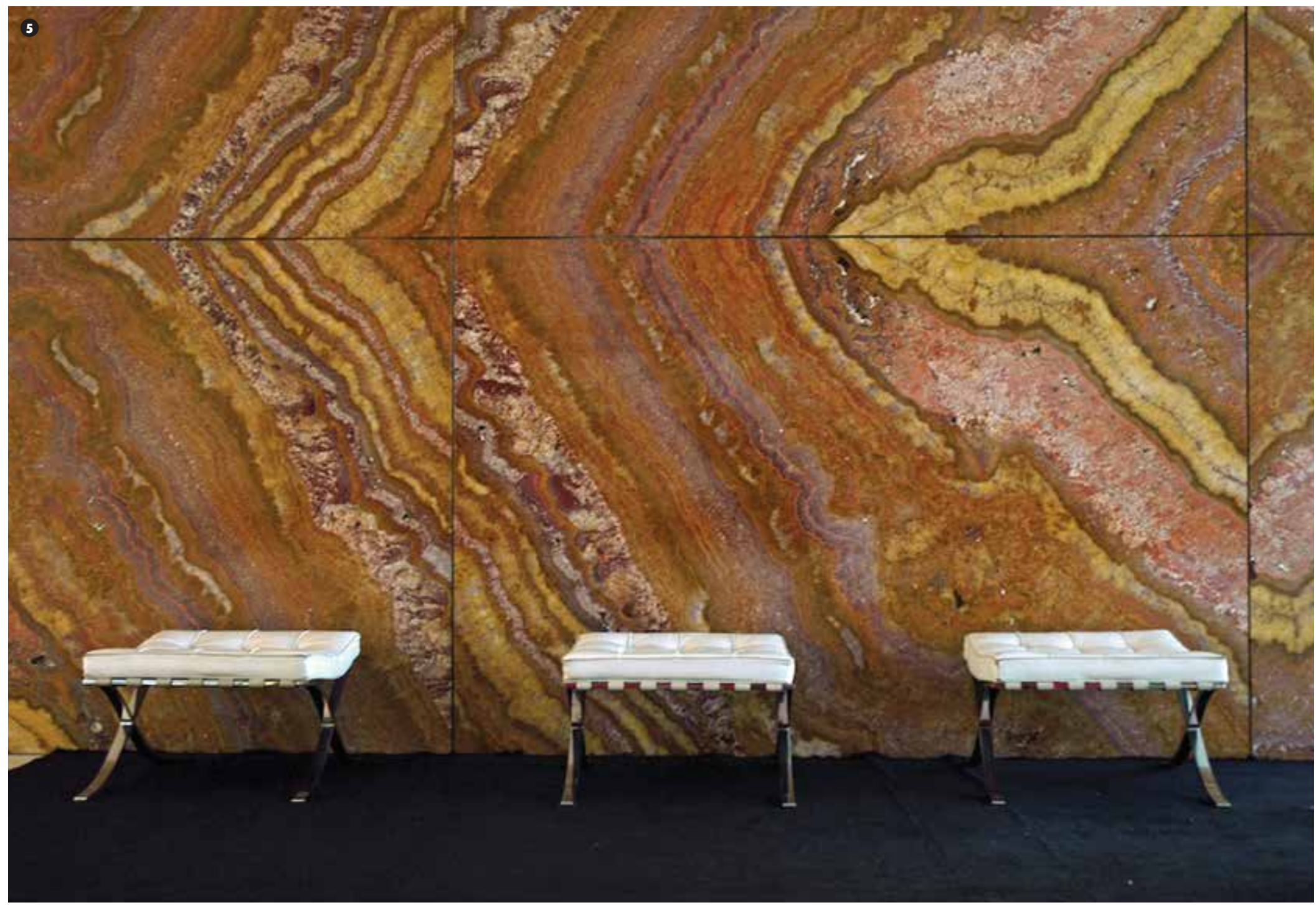

\section{EL ORNAMENTO COMO UNA NUEVA OPORTUNIDAD PARA LA ARQUITECTURA CONTEMPORÁNEA}

A comienzos del siglo xx, Adolf Loos condenó el uso del ornamento a nivel estético, económico y moral. Para él, el ornamento representaba el epítome de la bajeza humana, propia de las limitantes del hombre primitivo o de las degeneraciones del criminal. Su eliminación era el camino a la evolución de la sociedad y a la eficiencia de recursos. Sus palabras fueron muy escuchadas y el ornamento adquirió entre los arquitectos modernos la condición más deleznable posible. Desde entonces, y hasta hoy, ha estado vedado en la enseñanza de la arquitectura y se ha suprimido del léxico arquitectónico. El trauma que produjeron los excesos posmodernistas y sus intricadas invocaciones semióticas fue el tiro de gracia que terminó por sepultar al ornamento de la discusión disciplinar.

Aun así, no pudo ser erradicado. El ornamento y la subjetividad estética son inseparables al ser humano y está presente, aunque no se quiera, en todas sus obras. Está, por ejemplo, en la cuidadosa selección de veteados en los mármoles en el Pabellón de Alemania en Barcelona de Mies Van der Rohe o en la fina estampa de los moldajes en el hormigón de Le Corbusier. Está en las fachadas paramétricas, con o sin metáforas naturalistas, y con o sin simulaciones de control solar. La arbitrariedad y preferencias estéticas son lo que está detrás de los edificios singulares con estructuras desafiantes, no las particularidades técnicas de una máquina de fabricación digital. 
6. Pabellón Alemán en Barcelona, Mies van der Rohe Antonio Gallud
Hoy, después de un siglo, los argumentos de Loos se vuelven cuestionables. La eficiencia económica de una arquitectura sin ornamentación es rebatible en el contexto de tecnologías de manufactura y diseño digital que permiten automatizar procesos productivos sin aumentar mayormente los costos. La supuesta inmoralidad del ornamento y su concordancia con la degradación humana o con el retroceso social no resiste mayor análisis. Son los argumentos estéticos, amparados en la visión modernista funcionalista y positivista, los que se mantienen como el único obstáculo para que el ornamento vuelva a posicionarse con fuerza en los discursos arquitectónicos contemporáneos. Greg Lynn incipientemente planteó el problema a comienzos de la década; Farshid Moussavi y Michael Kubo entregaron su respuesta en el año 2006 validando su uso. Para ellos, el ornamento está asociado a los afectos estéticos, los que son tan aceptables como las consideraciones estructurales o funcionales. No son los únicos; Antoine Picon desde una perspectiva histórica y Robert Levit desde una crítica simbólica han reafirmado su retorno.

Parece oportuno entonces abrir hoy nuevas ventanas de reflexión en pos de entender -o eventualmente revalorizar- el rol que el ornamento contemporáneo debe cumplir en nuestra arquitectura. La tarea no es fácil: un siglo de veto y crítica no es en vano. Sin embargo, la pregunta está sobre la mesa, y la subjetividad y la arbitrariedad estética en medio del debate.

\section{BIBLIOGRAFÍA}

Levit, R. (2008). Contemporary Ornament: The Return of the Symbolic Repressed. Harvard Design Magazine, 28 (Spring/Summer), 70-85

Loos, A. (1972). Ornamento y delito, y otros ensayos. Barcelona: Gustavo Gili.

Lynn, G. (2004). The Structure of Ornament. En Leach et al., Digital Tectonics. Chichester: Wiley, 62-68. Moussavi, F. y Kubo, M. (2006). The Function of Ornament. Barcelona: Actar.

Pallasmaa J. (2005). The Eyes of the Skin: Architecture and the Senses. West Sussex: Wiley.

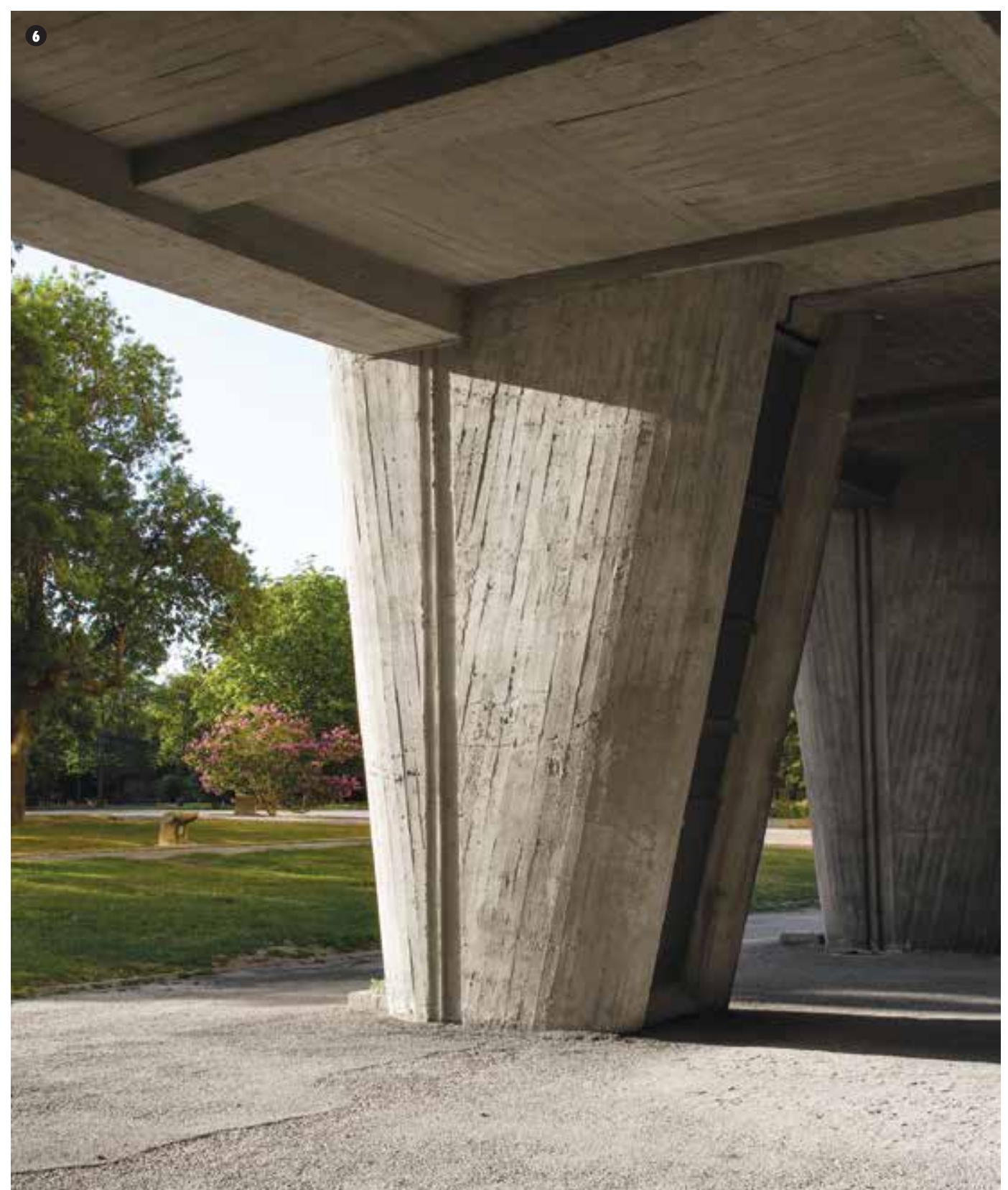

Picon, A. (2010). Digital culture in architecture. Birkhäuser.

Picon, A. (2013). Ornament: The Politics of Architecture and Subjectivity. Architectural Design Primer. New York: Wiley.

Schumacher, P. (2009). Parametricism: A new global style for architecture and urban design. Architectural Design, 79(4), 14-23.
Tschumi B (1996). Architecture and Disjunction. Cambridge: MIT Press.

Venturi, R., Brown, D. S., y Izenour, S. (1972). Learning from Las Vegas (Vol. 102). Cambridge, MA: MIT press. 